\title{
BMJ Open Design and evaluation of strategies to implement HIV prevention interventions for pregnant women in community pharmacy settings in western Kenya: a mixed-methods study protocol
}

\author{
Melissa Latigo Mugambi (10 , ${ }^{1}$ Jared M Baeten, ${ }^{1,2,3,4}$ John Kinuthia, ${ }^{1,5}$ Brett Hauber, ${ }^{6}$ \\ Bryan J Weiner, ${ }^{1,7}$ Grace John-Stewart, ${ }^{1,2,3}$ Ruanne Vanessa Barnabas ${ }^{1,3}$
}

To cite: Mugambi ML, Baeten JM, Kinuthia J, et al. Design and evaluation of strategies to implement HIV prevention interventions for pregnant women in community pharmacy settings in western Kenya: a mixed-methods study protocol. BMJ Open 2021;11:e052311. doi:10.1136/ bmjopen-2021-052311

- Prepublication history and additional supplemental material for this paper are available online. To view these files, please visit the journal online (http://dx.doi.org/10.1136/ bmjopen-2021-052311)

Received 27 April 2021 Accepted 22 November 2021

Check for updates

(C) Author(s) (or their employer(s)) 2021. Re-use permitted under CC BY-NC. No commercial re-use. See rights and permissions. Published by BMJ.

For numbered affiliations see end of article.

Correspondence to Dr Melissa Latigo Mugambi; mugambi@uw.edu

\begin{abstract}
Introduction Community pharmacies play an important role in the healthcare system: they are frequently accessed and have increasing capacity to deliver HIV prevention services. In communities where the prevalence of HIV is high and access to antenatal care clinics is delayed or irregular, there is a unique opportunity to leverage pharmacies to enhance early and sustained access to HIV prevention among pregnant women. This study will identify women's preferences for delivery of HIV prevention services and provider-level and system-level strategies to design a new pharmacy-based model of care for pregnant women. The overall objective of this study is to design and evaluate strategies to implement HIV prevention interventions for pregnant women in community pharmacy settings in western Kenya.
\end{abstract}

Methods and analysis We propose to conduct a discrete choice experiment to quantify preferences for delivery of HIV prevention interventions (including pre-exposure prophylaxis, partner testing and sexually transmitted infection screening and treatment) for pregnant women in community pharmacy settings. Latent class analysis will be used to quantify women's stated preferences and identify packages of intervention components that will optimise uptake among different subgroups of women. We will apply the Theoretical Domains Framework to identify provider-level and system-level factors that might influence the implementation of the optimal intervention packages. We will then use the Behaviour Change Wheel and survey a panel of experts to select and gain consensus on strategies to improve implementation. Finally, we will evaluate the potential costs of extending the implementation of HIV prevention interventions from the clinic to community pharmacy settings.

Ethics and dissemination The protocol was approved by the Kenyatta National Hospital-University of Nairobi Ethics Research Committee and the University of Washington Institutional Review Board. The results of this research will be published in peer-reviewed journals and shared with various stakeholders, including community members, policymakers and researchers, through local and international conferences.
Strengths and limitations of this study

- This study will qualitatively identify and understand multiple stakeholder (including users, providers and decision-makers) perceptions and priorities of potential features of a pharmacy-delivered HIV prevention intervention package for pregnant women.

- In the discrete choice experiment, the study will systematically and quantitatively tailor and optimise the design of an intervention based on potential user preferences before implementation and account for preference heterogeneity among potential users.

- This study will apply the Theoretical Domains Framework and Behaviour Change Wheel to systematically and theoretically identify potential barriers to implementing a pharmacy-delivered HIV prevention package and design strategies to overcome the barriers.

- The Delphi survey and costing evaluation will methodologically establish expert consensus on priority and feasible implementation strategies.

- Potential limitations include leveraging data from rural and urban populations in western Kenya that might not apply to other settings and the general assumption that what potential users state about the intervention will reflect actual utilisation.

\section{INTRODUCTION}

In high HIV-burden settings such as western Kenya, the risk of HIV acquisition among pregnant women is two to three times higher than non-pregnant/non-postpartum women. Furthermore, the risk of mother-to-child transmission is two to three times higher among women who acquire HIV during pregnancy than those with chronic infection. ${ }^{1}$ The WHO and Ministry of Health in Kenya recommend a comprehensive HIV prevention package for pregnant women in 
high HIV-burden settings, including risk assessment, HIV pre-exposure prophylaxis (PrEP) for those at substantial risk, sexually transmitted infection (STI) screening and treatment and partner testing and treatment. ${ }^{2}$ However, delayed and irregular utilisation of antenatal care limits opportunities to realise the full benefits of HIV prevention services. ${ }^{3}$ For example, in western Kenya, only one in five women present for antenatal care within the first trimester, and just over half of the women attend four or more antenatal care visits. ${ }^{4}$ While more than $90 \%$ of pregnant women are tested for HIV, three in five pregnant women do not receive a repeat HIV test before delivery. ${ }^{56}$ Partner testing rates also remain low in many sub-Saharan African countries. ${ }^{7}$ Whereas facility-level barriers to early antenatal care have been well-documented, including high transportation costs, long travel distances and lengthy clinic wait times, ${ }^{8}$ few interventions have successfully addressed delayed initiation of antenatal care. ${ }^{9}$ To optimise the public health impact of HIV prevention interventions, pregnant women at substantial risk for HIV infection must successfully navigate the antenatal care and HIV prevention pathway, including being identified early, engaged and retained in care. Closing this gap will be critical in complementing ongoing efforts to determine the best model of delivering PrEP in routine public-sector antenatal care clinics. ${ }^{10}$

In settings like Kenya, community pharmacies are often the first point of contact for basic health services, ${ }^{11}$ and might be strategically positioned to address facility-level barriers to early antenatal care. Compared with health facilities, pharmacies are more accessible, save time, provide information on how to use health products and demonstrate high consumer demand for various sexual and reproductive health products. ${ }^{12}$ For example, nearly half of the women who use oral contraceptive pills obtain them from pharmacies. ${ }^{4}$ Additionally, our preliminary data show that four in five women who use pregnancy selftests purchase them from community pharmacies. In a community survey, over one-third of the women expressed interest in obtaining PrEP via community pharmacies. ${ }^{13} 14$ Within the antenatal care context, pharmacy providers already educate and refer pregnant women for antenatal care in early pregnancy. ${ }^{15}$ In Uganda, using pharmacies to deliver malaria prevention services for pregnant women has been feasible and has increased the early uptake of services. ${ }^{16}$ Within the HIV context, pharmacies have successfully been employed to improve uptake of antiretroviral therapy in resource-limited settings and PrEP in resource-rich settings. ${ }^{17}$ Leveraging existing structures that are frequently accessed, such as community pharmacies, might create cost-efficient opportunities for pregnant women to initiate antenatal care and HIV prevention early. There is a unique opportunity to implement HIV prevention interventions for pregnant women in community pharmacy settings. However, it is unclear which package of interventions would best satisfy pregnant women's needs and optimise uptake, which strategies should be used for implementation of these interventions, and the cost of extending these interventions to community pharmacy settings.

Therefore, the proposed research aims to close gaps in the perinatal HIV prevention cascade by designing strategies for implementing HIV prevention interventions in community pharmacies where pregnant women at high risk for HIV can be identified. ${ }^{12}$

\section{METHODS AND ANALYSIS}

Figure 1 summarises the research strategy and methods applied in this study. The overall objective of this study is to

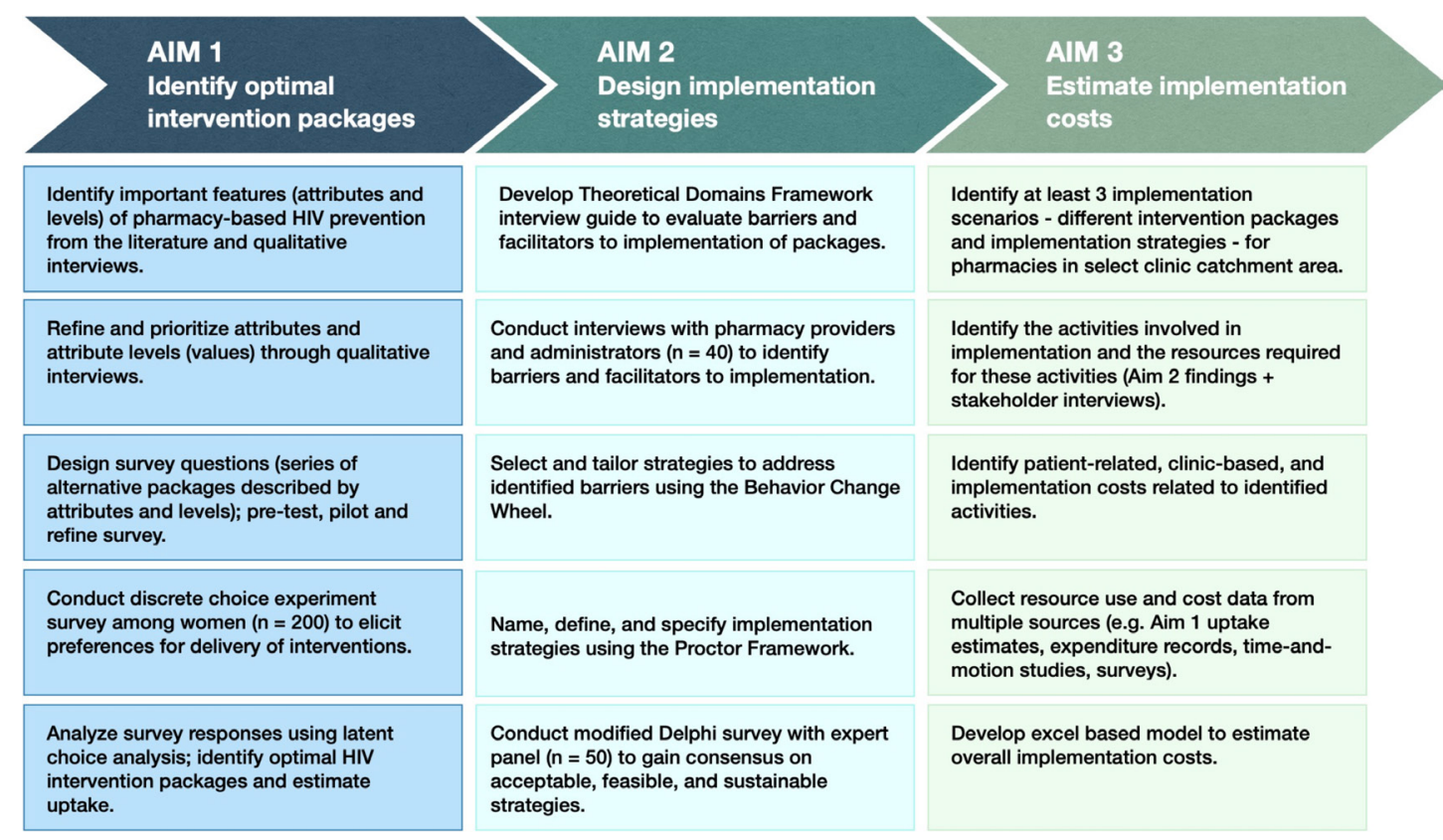

Figure 1 Overall research strategy and methods. 


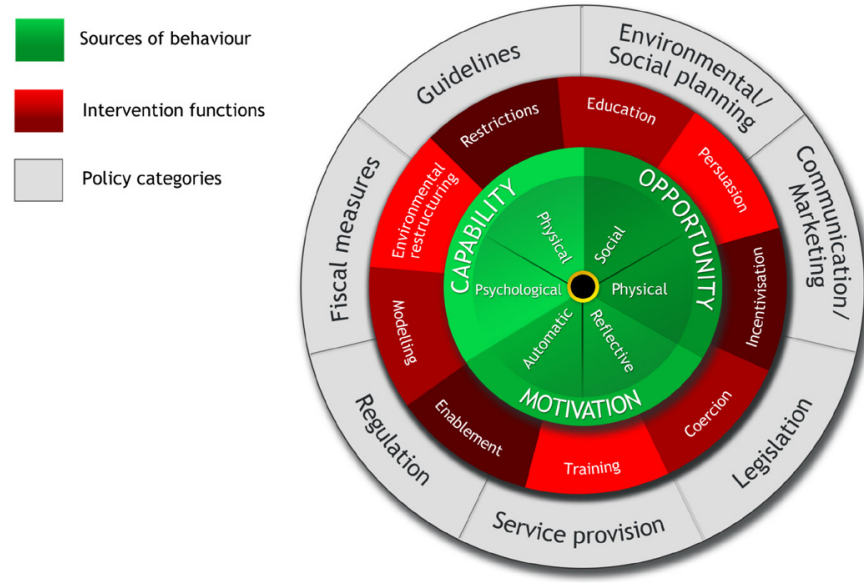

Figure 2 Behaviour Change Wheel reproduced from Michie et al. ${ }^{19}$

design and evaluate strategies to implement HIV prevention interventions for pregnant women in community pharmacy settings. To accomplish this objective, we will use implementation science theory drawing on a range of novel and established methods (determinant frameworks and theories, discrete choice experiments (DCEs) and ingredients-based costing) to elicit preferences for intervention delivery, identify implementation strategies and estimate their costs. Our approach advances previous approaches by allowing for variability in how and where HIV prevention interventions are offered versus assuming that uptake is homogeneous and takes place at the clinic only. ${ }^{18}$ The specific objectives are:

1. To quantify preferences for delivery of HIV prevention interventions for pregnant women in community pharmacy settings.

2. To identify the factors that may influence the implementation of HIV prevention interventions for pregnant women in community pharmacy settings and select strategies to improve implementation.
3. To estimate the costs of extending implementation of HIV prevention interventions from the clinic to community pharmacy settings.

\section{Conceptual framework}

We will apply the Theoretical Domains Framework (TDF) and Behaviour Change Wheel (BCW) in Objective 2 (figures 2 and 3, reproduced from Michie et al. ${ }^{19}$ ). The TDF is an original framework for implementation science synthesising 33 behaviour change and implementation theories into 14 domains that describe determinants of behaviour. These 14 domains can further be synthesised into three major domains representing the capability, opportunity and motivation for a particular behaviour. These three domains form the hub of the BCW and are mapped to broad categories of implementation strategies that can target barriers to behaviour (intervention functions and behaviour change techniques) which in turn are mapped to approaches that can be used to deliver proposed strategies (policy categories). ${ }^{19}$ Finally, the APEASE criteria (affordability, practicability, effectiveness/cost-effectiveness, acceptability, side-effects/safety and equality) can be used to identify contextually relevant strategies. ${ }^{20}$ The BCW therefore provides an approach to identify potential barriers to behaviour change and the strategies to overcome the barriers in a given context. In Objective 2, we will first use the TDF to create an interview guide to identify potential provider-level and system-level barriers to implementing HIV prevention interventions in community pharmacies. Subsequently, we will use the BCW to identify potential implementation strategies that might overcome the identified barriers.

\section{Objective 1: discrete choice experiment}

Our objective is to identify optimal packages of HIV prevention interventions and options for delivery that would best satisfy pregnant women's needs in community pharmacy settings. We ask women of reproductive age to make choices among different hypothetical

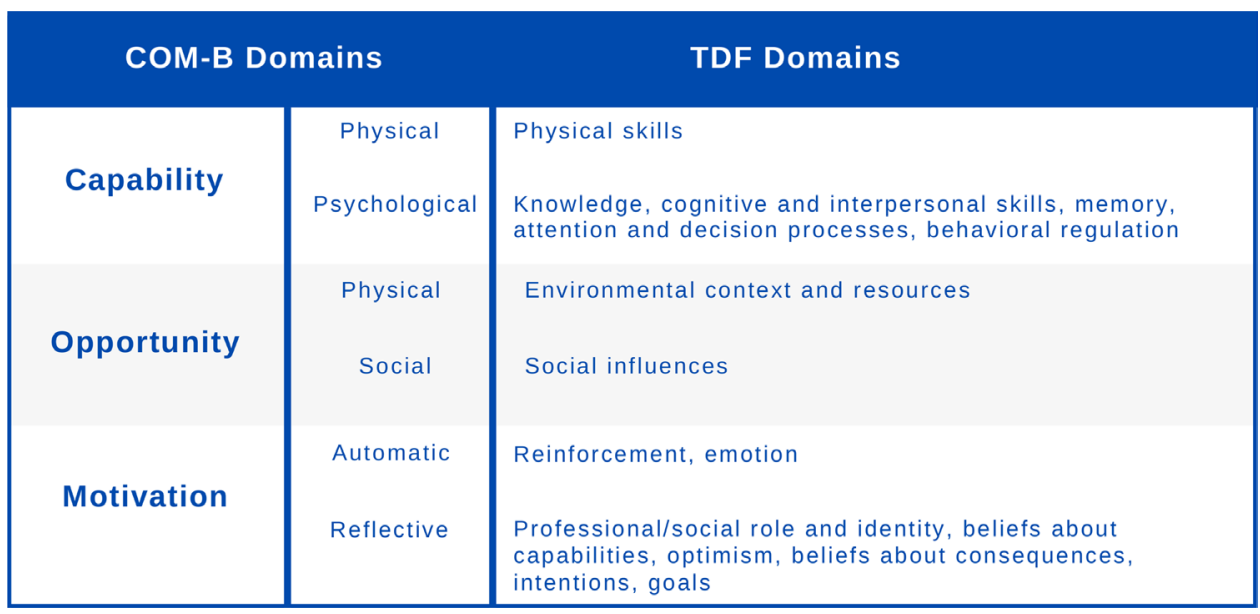

Figure 3 The Theoretical Domains Framework (TDF) in relation to the capability, opportunity and motivation behaviour (COM-B) model. 


\begin{tabular}{ll} 
Table 1 Potential attributes and levels \\
\hline Attribute & Level \\
\hline Location & Pharmacy. \\
Type of initial service & Clinic. \\
provider & Pharse. \\
HIV testing & Pharmacist. \\
& HIV self-testing, counselling and \\
& referral to ANC clinic for additional \\
& HIV prevention services. \\
& HIV self-testing, counselling, HIV \\
& receive prevention services. \\
& PrEP not available. \\
& PrEP dispensing and refills \\
& available. \\
PrEP & PrEP refills only. \\
& STI management (screening and \\
& testing). \\
& $>$
\end{tabular}

ANC, antenatal care; PrEP, pre-exposure prophylaxis; STI, sexually transmitted infection.

HIV prevention interventions and delivery options for community pharmacy settings. We present respondents with a series of choice questions that describe two or more package alternatives. Each alternative is composed of a set of attributes, each with different levels where, for example, 'location' is an attribute and 'pharmacy' or 'clinic' is a level (table 1 ).

In choosing the preferred package, respondents make tradeoffs between different attribute levels based on the value they place on the different levels (eg, assuming 'PrEP' is another attribute with the levels 'PrEP dispensing and refills available' and 'PrEP not available', would they select to receive PrEP at the pharmacy or clinic if available in both settings). We then use choice modelling methods to quantify preferences for attribute levels— that is-estimate the marginal or part-worth utility attached to each attribute level, and determine choice probabilities (the likelihood of uptake) among the respondents. DCEs have been recommended as a systematic and robust approach for tailoring interventions before implementation. ${ }^{21}$

\section{Selection of attributes and levels}

We will begin by identifying potential attributes and levels from preliminary qualitative data and the literature. ${ }^{22} \mathrm{We}$ will conduct up to five focus group discussions (FGDs) with women $(\mathrm{n}=5-10)$ and five FGDs with health providers $(\mathrm{n}=5-10$ community health workers and nurses), and five semi-structured interviews with decision-makers to further refine and identify additional attributes. We primarily want to assess whether women would consider going to a pharmacy instead of an antenatal care clinic during pregnancy for HIV prevention products or services. Therefore, interview topics will include barriers and facilitators to uptake of HIV prevention products or services in pharmacies and antenatal care clinics, product and service delivery preferences, and attribute importance and rationale (see online supplemental file 1 for more details). Interview transcripts will be audio-recorded, transcribed, translated to English as needed and coded independently by at least two researchers to identify important attributes and levels. In a second round of FGDs and interviews (as described above), we will narrow down the attributes to focus on four to six of the most influential ones by conducting a card sorting exercise in which stakeholders select and rank a set of cards labelled with different potential attributes. Stakeholders will also provide input on adequate level ranges. We can use the attributes and levels identified to generate a series of package alternatives with all possible combinations of attributes and levels, also known as a full factorial design. However, this would impose a huge respondent burden, and some combinations might be infeasible. Therefore, we will only select a sample of these combinations (a fractional factorial design) using Sawtooth Software. For each choice question, we will select combinations with independent attributes and minimal overlap of attribute levels so that respondents can differentiate among the package alternatives.

\section{Survey design}

We will design the survey instrument to include various sections for collecting data on sociodemographic characteristics, knowledge and experiences with antenatal care and HIV prevention interventions, pharmacy and clinic-based care, costs and respondent choices to the DCE. In the DCE section, respondents will answer a series of choice questions (table 2); we will include an opt-out or 'neither' alternative to determine the likelihood of uptake of different packages.

The survey instrument will be translated into the local languages (Swahili and Dholuo); at least two translators will independently back-translate the questionnaires to verify that the translation is consistent with the original content. We will pre-test and pilot the survey instrument on up to 30 women to identify potential areas for refinement (eg, attribute definitions, level ranges, translation adequacy). The pilot will also help us identify the maximum number of choice questions to which participants can respond without becoming fatigued-typically around 9-16 questions. This number will inform the number of survey versions (eg, if there are 40 questions and the maximum number that respondents can respond to is eight, there will be five versions of the survey). One version will be randomly assigned to each respondent. Surveys will be conducted via face-to-face interviews. We will make every effort to minimise hypothetical bias (differences between 
Table 2 Sample choice question

\begin{tabular}{|c|c|}
\hline Package 1 & Package 2 \\
\hline Go to pharmacy & Go to clinic \\
\hline $\begin{array}{l}\text { Seen by pharmacist with } \\
\text { nurse oversight }\end{array}$ & Seen by nurse \\
\hline $\begin{array}{l}\text { Conduct HIV self-test, } \\
\text { receive counselling and } \\
\text { HIV risk assessment with } \\
\text { option to receive prevention } \\
\text { services on site }\end{array}$ & $\begin{array}{l}\text { Conduct HIV self-test, receive } \\
\text { counselling and HIV risk } \\
\text { assessment with option to } \\
\text { receive prevention services } \\
\text { on site }\end{array}$ \\
\hline PrEP not available & $\begin{array}{l}\text { PrEP dispensing and refills } \\
\text { available }\end{array}$ \\
\hline $\begin{array}{l}\text { STI management and partner } \\
\text { testing available }\end{array}$ & $\begin{array}{l}\text { STI management and partner } \\
\text { testing available }\end{array}$ \\
\hline Total time spent: $30 \mathrm{~min}$ & Total time spent: 3 hours \\
\hline \multicolumn{2}{|c|}{$\begin{array}{l}\text { Which of these two packages would you choose? } \\
\square \text { Package } 1 \square \text { Package } 2 \square \text { Neither }\end{array}$} \\
\hline
\end{tabular}

PrEP, pre-exposure prophylaxis; STI, sexually transmitted infection.

actual and stated choices) by building multiple validation tests into the survey and analysis. ${ }^{23}$

\section{Study setting and population}

We plan to conduct the study in western Kenya. Our target population of interest includes women of childbearing age (15-44 years of age) who are HIV negative, actual or potential users of community pharmacies, and able and willing to provide informed consent for participation. Women who are unemancipated minors by Kenyan law (ie, younger than 18 years of age and have not been pregnant) will not be eligible to participate in the study. Women who participate in the initial FGDs and DCE survey pilot will not be eligible to participate in the final DCE survey. We will recruit a simple random sample of participants $(n=200)$ from ongoing PrEP implementation studies in antenatal care and pharmacy settings. ${ }^{22} 24$ Women will be recruited using various methods including poster displays that encourage customers to ask their pharmacy provider about antenatal care services and having pharmacy providers ask customers buying pregnancy tests if they are interested in participating in the study. In antenatal care clinics, study staff will identify potential participants who meet inclusion criteria during routine appointments or through follow-up phone calls and invite them to participate in the study. Potential study participants will be given a referral letter that includes information on how the participant was identified, the purpose of the study and a brief description of the activities in which they will participate.

\section{Sample size}

There is little consensus on calculating the minimum sample size required to obtain adequate statistical power to test hypotheses in DCE studies. A general principle suggests a minimum sample size of between 250 and 350 respondents for robust quantitative research. However, this approach is not necessarily theoretical and is based on experience from a small subset of studies. ${ }^{25}{ }^{26}$ When we apply an equation derived for simple random samples that factors the true choice proportion of the relevant population $(10 \%)$, confidence $(95 \%)$ and precision level $(10 \%)$, and the number of choice questions (12), the desired sample size is $147 .{ }^{25}$ In this study, we take a conservative approach by factoring in the various approaches used to calculate sample size and therefore aim to enrol a minimum of 200 women in the DCE survey.

\section{Data analysis}

We will use latent class models to analyse the preference data generated from the DCE survey. ${ }^{27}$ We will estimate the preference weight or part-worth utility attached to each attribute level and choice probabilities (likelihood of uptake). In additional analyses, we will assess the extent to which classes or subgroups with systematically different preference weights exist in the sample and the types of individuals that are more likely to make certain choices. To identify optimal packages of HIV prevention interventions, we will review the DCE findings and identify attributes that have strong preference weights and are feasible to implement.

\section{Objective 2: qualitative interviews and Delphi survey}

Our objective is to identify potential barriers and facilitators to implementing the optimal packages of HIV prevention interventions identified in Objective 1. Guided by the TDF, we will identify and prioritise provider-level and system-level barriers and facilitators to implementation. ${ }^{28}$ Successful completion of this objective is expected to contribute to a better understanding of factors that might influence the implementation of HIV prevention interventions and design strategies to implement these interventions in community pharmacy settings.

\section{Study setting and population}

We will interview up to 40 community pharmacists and administrators from a sample of community pharmacies selected based on criteria that may influence the perception of the intervention and effectiveness of the implementation strategy, including geographical location (urban vs rural), affiliation (franchise vs stand-alone) and size. We will leverage ongoing studies to identify potential community pharmacies for inclusion in the study. ${ }^{22}$

\section{Study design and data collection}

Qualitative data will be collected through individual interviews using an interview guide based on TDF constructs. As ascertained in prior studies, we will assess select constructs from the TDF based on their likely influence on implementation. ${ }^{22}{ }^{29}$ Interview sessions will last $60 \mathrm{~min}$ and focus on discussion topics guided by select constructs; we will also ask open-ended questions to explore the possibility of other constructs and their influences on implementation. Interviews will be conducted in English and Swahili, audio-recorded and transcribed and translated into English as needed. We will prepare analytical 
memos to describe the communication and behaviour of respondents and the interviewers' reflections during the data collection process.

\section{Qualitative analysis}

ATLAS.ti software will be used to support review and coding of the transcripts. Guided by the TDF constructs, at least two researchers will carefully review the transcripts to identify important concepts or 'codes'. ${ }^{28}$ A few transcripts will also be reviewed using open coding techniques to ensure that important concepts are not missed. An agreed-upon set of codes will be categorised (eg, by provider-level and system-level barriers), defined and used to code subsequent transcripts. To establish interrater reliability, at least two researchers will independently code each transcript, compare their coding and reconcile coding discrepancies. To guide data analysis, we will use the framework method, a type of thematic analysis that summarises themes on barriers and facilitators in a spreadsheet matrix by case (eg, type of pharmacy) and by code. ${ }^{30}$ We will explore how these themes vary across different types of pharmacies and within the same types of pharmacies and assess their importance based on the frequency of occurrence and likelihood of impact on implementation.

\section{Selection of implementation strategies}

We will systematically analyse the barriers and facilitators to identify potential behaviour change targets and select corresponding implementation strategies using the BCW. ${ }^{19}$ We will use Proctor's criteria for specifying and reporting implementation strategies to identify the actors involved, specific activities and the timing and frequency of these activities. ${ }^{31}$ We will identify and achieve consensus on priority and contextually relevant implementation strategies via a modified Delphi technique using the criteria for the reporting of Delphi studies. ${ }^{32}$ A purposefully recruited expert panel $(\mathrm{n}=50$, assuming an attrition rate of $50 \%$ ) will participate in two or more rounds of surveys to gain consensus ( $>80 \%$ with the same rating) on the implementation strategies. The APEASE criteria will guide strategy selection. ${ }^{19}$

\section{Objective 3: cost analysis}

Our objective is to explore the cost implications of extending the implementation of user-preferred HIV prevention interventions to community pharmacies. Increasing options for HIV prevention delivery can increase the number of pregnant women who initiate and continue using prevention interventions and result in potential cost-efficiencies by reducing demands on the clinic. However, increased referrals from the pharmacy to the clinic or the need to invest in improving the quality of pharmacy-based delivery could also increase programmatic costs. At the patient level, the community pharmacy can provide a more convenient option for accessing care and reduce the costs associated with transportation and wait times; however, patients might also incur out-of-pocket costs. Evaluating costs before implementation plays an essential role in planning and refining future implementation efforts and guiding investment decisions. ${ }^{33}$ Cost implications and estimates will help identify and prioritise potential implementation strategies that can be tested empirically and evaluated for costeffectiveness in a future study.

\section{Approach}

Costs will be determined from the 'limited societal perspective' which includes the perspective of the decision-makers paying for implementation and select patient costs. In addition to leveraging our findings on implementation activities from Objective 2, we will interview up to 10 stakeholders (decision-makers and individuals who have been involved in similar implementation activities) to identify and prioritise implementationrelated costs and impacts; findings will inform the type of cost data that is collected. We will estimate costs using an ingredients-based approach. Costs will be calculated in US dollars, and adjusted for inflation. Cost estimates will be projected over a 1-year period therefore costs will not be discounted. Cost analyses will be modelled in Excel. To guide our overall approach, we will estimate the cost of extending implementation to community pharmacies within a select clinical catchment area. We will consider at least three implementation scenarios under a range of different intervention packages (Objective 1) and implementation strategies (Objective 2).

\section{Data collection and analysis}

We anticipate estimating costs from three broad cost categories:

1. The costs associated with implementing HIV prevention interventions in pharmacy settings under a range of different strategies identified in Objective 2 (implementation costs).

2. The costs associated with potential healthcare resource utilisation changes in the clinical setting (clinicalbased costs).

3. Patient-related costs.

We will use the choice (uptake) probabilities from Objective 1 to determine the likelihood of women using services at the pharmacy or clinic. Overall, we will estimate the total costs of delivering HIV prevention services via the clinic only (base case); for each implementation scenario, we will estimate the total costs of extending pregnancy-related HIV prevention services to the community pharmacy setting and the cost per individual reached. To test the robustness of our cost estimates, we will conduct a sensitivity analysis around key resource inputs and assumptions influencing the quantity or value of these inputs.

\section{Patient and public involvement}

The proposed study will leverage expertise and resources from several ongoing PrEP implementation studies in antenatal care and pharmacy settings. ${ }^{22}{ }^{24}$ The studies 
have community and stakeholder advisory boards that will provide opportunities to share study queries and findings with community members, leaders and decisionmakers. In June 2019, we shared preliminary research objectives with the community advisory board members and obtained their input on the feasibility of leveraging community pharmacies to identify pregnant women who are at risk for HIV acquisition and can benefit from PrEP. Key feedback received, which will be helpful as we think through the design of the prevention package and pharmacy implementation strategy, include the need for community health worker engagement and training, provision of nurse or physician oversite and adolescent engagement due to increases in pregnancy among this population. We plan to periodically share study findings with the advisory board members as well as other community and policy stakeholders in local and international meetings.

\section{Timeline}

We anticipate beginning data collection activities in Q1 of 2022 and finalising data collection and analysis in Q4 of 2024 .

\section{ETHICS AND DISSEMINATION}

The protocol was approved by the Kenyatta National Hospital-University of Nairobi Ethics Research Committee and the University of Washington Institutional Review Board. The results of this research will be published in peer-reviewed journals and shared with various stakeholders, including community members, researchers and policy-makers, through local and international conferences. In a future pilot study, we will evaluate the acceptability, feasibility and impact of optimal intervention packages and implementation strategies identified in Objectives 1 and 2. We will use the cost data generated in Objective 3 to plan for implementation and evaluate the cost-effectiveness of piloted strategies.

\section{Author affiliations}

${ }^{1}$ Department of Global Health, University of Washington, Seattle, Washington, USA ${ }^{2}$ Department of Epidemiology, University of Washington, Seattle, Washington, USA ${ }^{3}$ Department of Medicine, University of Washington, Seattle, Washington, USA ${ }^{4}$ Gilead Sciences, Foster City, California, USA

${ }^{5}$ Department of Research and Programs, Kenyatta National Hospital, Nairobi, Kenya ${ }^{6}$ The Comparative Health Outcomes, Policy and Economics (CHOICE) Institute, Department of Pharmacy, University of Washington, Seattle, Washington, USA ${ }^{7}$ Department of Health Systems and Population Health, University of Washington, Seattle, Washington, USA

Acknowledgements We acknowledge the PrEP Implementation for Mothers in Antenatal Care (PrIMA) study team including Jillian Pintye, Anjuli Wagner, Nancy Mwongeli, Emmaculate Mukenyi and Rita Akumu for their feedback and support in preparing the grant and Institutional Review Board applications. We acknowledge Joseph Babigumira and Carol Levine for their comments on the grant application that were instrumental in preparing this study protocol. We acknowledge the HIV, Infectious Disease, and Global Health Implementation Research Institute (HIGH IRI) for the mentorship support provided toward this study. Finally, we acknowledge the community members that will participate in this study.

Contributors This study was conceptualised by MLM (principal investigator) with input from JMB, JK, BH, GJ-S, BJW and RVB who all serve on MLM's research mentoring committee. The first draft of this study protocol was written by MLM. All authors reviewed, edited and approved the study protocol.

Funding This work was funded by the National Institutes of Mental Health (NIMH), grant number 1K01MH122326-01A1.

Competing interests RVB reports grants from King K. Holmes Professorship in STDs and AIDS, Bill and Melinda Gates Foundation (BMGF), National Institutes of Health $(\mathrm{NIH})$ and manuscript and abstract writing support from Regeneron Pharmaceuticals outside the submitted work. BH reports stock options from Pfizer and grants from NIH outside the submitted work. BH is a paid employee at Pfizer. JMB reports personal fees from Gilead Sciences, Janssen and Merck, and grants from NIH, CDC, USAID and BMGF, outside the submitted work. JMB is a paid employee at Gilead Sciences. GJ-S reports grants from NIH, CDC, Thrasher Foundation, IMPAACT and stock options from the Malaika HIV vaccine outside the submitted work. GJ-S sits on the DSMB of the VITALITY and Tatelo trials.

Patient consent for publication Not applicable.

Provenance and peer review Not commissioned; externally peer reviewed.

Supplemental material This content has been supplied by the author(s). It has not been vetted by BMJ Publishing Group Limited (BMJ) and may not have been peer-reviewed. Any opinions or recommendations discussed are solely those of the author(s) and are not endorsed by BMJ. BMJ disclaims all liability and responsibility arising from any reliance placed on the content. Where the content includes any translated material, BMJ does not warrant the accuracy and reliability of the translations (including but not limited to local regulations, clinical guidelines, terminology, drug names and drug dosages), and is not responsible for any error and/or omissions arising from translation and adaptation or otherwise.

Open access This is an open access article distributed in accordance with the Creative Commons Attribution Non Commercial (CC BY-NC 4.0) license, which permits others to distribute, remix, adapt, build upon this work non-commercially, and license their derivative works on different terms, provided the original work is properly cited, appropriate credit is given, any changes made indicated, and the use is non-commercial. See: http://creativecommons.org/licenses/by-nc/4.0/.

ORCID iD

Melissa Latigo Mugambi http://orcid.org/0000-0001-8875-5528

\section{REFERENCES}

1 Thomson KA, Hughes J, Baeten JM, et al. Increased risk of HIV acquisition among women throughout pregnancy and during the postpartum period: a prospective Per-Coital-Act analysis among women with HIV-infected partners. J Infect Dis 2018;218:16-25.

2 WHO. Preventing HIV during pregnancy and breastfeeding in the context of PreP. Geneva, Switzerland: World Health Organization, 2017.

3 Rogers AJ, Weke E, Kwena Z, et al. Implementation of repeat HIV testing during pregnancy in Kenya: a qualitative study. BMC Pregnancy Childbirth 2016;16:151.

4 KNBS. Kenya demographic and health survey, 2014. Calverton, MD: Kenya National Bureau of Standards, 2014

5 Rogers AJ, Akama E, Weke E, et al. Implementation of repeat HIV testing during pregnancy in southwestern Kenya: progress and missed opportunities. J Int AIDS Soc 2017;20:e25036.

6 Sirengo M, Muthoni L, Kellogg TA, et al. Mother-To-Child transmission of HIV in Kenya: results from a nationally representative study. J Acquir Immune Defic Syndr 2014;66 Suppl 1:S66.

7 Manjate Cuco RM, Munguambe K, Bique Osman N, et al. Male partners' involvement in prevention of mother-to-child HIV transmission in sub-Saharan Africa: a systematic review. Sahara J 2015;12:87-105.

8 Pell C, Meñaca A, Were F, et al. Factors affecting antenatal care attendance: results from qualitative studies in Ghana, Kenya and Malawi. PLoS One 2013;8:e53747.

9 Mbuagbaw L, Medley N, Darzi AJ, et al. Health system and community level interventions for improving antenatal care coverage and health outcomes. Cochrane Database Syst Rev 2015;12:CD010994.

10 Kinuthia J, Pintye J, Abuna F, et al. Pre-Exposure prophylaxis uptake and early continuation among pregnant and post-partum women within maternal and child health clinics in Kenya: results from an implementation programme. Lancet HIV 2020;7:e38-48.

11 Wafula FN, Miriti EM, Goodman CA. Examining characteristics, knowledge and regulatory practices of specialized drug shops in 
sub-Saharan Africa: a systematic review of the literature. BMC Health Serv Res 2012;12:223.

12 Corroon M, Kebede E, Spektor G, et al. Key role of drug shops and pharmacies for family planning in urban Nigeria and Kenya. Glob Health Sci Pract 2016;4:594-609.

13 Begnel ER, Escudero J, Mugambi M, et al. High pre-exposure prophylaxis awareness and willingness to pay for pre-exposure prophylaxis among young adults in Western Kenya: results from a population-based survey. Int J STD AIDS 2020;31:454-9.

14 Nganga N, Dettinger J, Kinuthia J, et al. Prevalence and correlates of pregnancy self-testing among pregnant women attending antenatal care in Western Kenya. PLoS One 2021;16:e0258578.

15 Dillip A, Kimatta S, Embrey M, et al. Can formalizing links among community health workers, accredited drug dispensing outlet dispensers, and health facility staff increase their collaboration to improve prompt access to maternal and child care? A qualitative study in Tanzania. BMC Health Serv Res 2017;17:416.

16 Mbonye AK, Magnussen P, Bygbjerg IB. Intermittent preventive treatment of malaria in pregnancy: the effect of new delivery approaches on access and compliance rates in Uganda. Trop Med Int Health 2007;12:519-31.

17 Tung EL, Thomas A, Eichner A, et al. Implementation of a community pharmacy-based pre-exposure prophylaxis service: a novel model for pre-exposure prophylaxis care. Sex Health 2018;15:556-61.

18 Roberts DA, Barnabas RV, Abuna F, et al. The role of costing in the introduction and scale-up of HIV pre-exposure prophylaxis: evidence from integrating PreP into routine maternal and child health and family planning clinics in Western Kenya. J Int AIDS Soc 2019;22 Suppl 4:e25296.

19 Michie S, van Stralen MM, West R. The behaviour change wheel: a new method for characterising and designing behaviour change interventions. Implement Sci 2011;6:42.

20 Michie S, Atkins L, West R. The behaviour change wheel: a guide to designing interventions. Needed: physician leaders 2014;26:146.

21 Louviere JJ, Hensher DA, Swait JD. Stated choice methods: analysis and applications: Cambridge university press, 2000.
22 Ortblad KF, Mogere P, Roche S, et al. Design of a care pathway for pharmacy-based PreP delivery in Kenya: results from a collaborative stakeholder consultation. BMC Health Serv Res 2020;20:1-9.

23 Janssen EM, Marshall DA, Hauber AB, et al. Improving the quality of discrete-choice experiments in health: how can we assess validity and reliability? Expert Rev Pharmacoecon Outcomes Res 2017; $17: 531-42$

24 Dettinger JC, Kinuthia J, Pintye J, et al. Prep implementation for mothers in antenatal care (PRiMA): study protocol of a cluster randomised trial. BMJ Open 2019;9:e025122.

25 Rose JM, Bliemer MCJ. Sample size requirements for stated choice experiments. Transportation 2013;40:1021-41.

26 Yang J-C, Johnson FR, Kilambi V, et al. Sample size and utilitydifference precision in discrete-choice experiments: a metasimulation approach. J Choice Model 2015;16:50-7.

27 Hauber AB, González JM, Groothuis-Oudshoorn CGM, et al. Statistical methods for the analysis of discrete choice experiments: a report of the ISPOR conjoint analysis good research practices Task force. Value Health 2016;19:300-15.

28 Atkins L, Francis J, Islam R, et al. A guide to using the theoretical domains framework of behaviour change to investigate implementation problems. Implement Sci 2017;12:77.

29 Roche SD, Wairimu N, Mogere P, et al. Acceptability and feasibility of Pharmacy-Based delivery of pre-exposure prophylaxis in Kenya: a qualitative study of client and provider perspectives. AIDS Behav 2021;25:3871-82.

30 Gale NK, Heath G, Cameron E, et al. Using the framework method for the analysis of qualitative data in multi-disciplinary health research. BMC Med Res Methodol 2013;13:117.

31 Proctor EK, Powell BJ, McMillen JC. Implementation strategies: recommendations for specifying and reporting. Implement Sci 2013;8:139.

32 Diamond IR, Grant RC, Feldman BM, et al. Defining consensus: a systematic review recommends methodologic criteria for reporting of Delphi studies. J Clin Epidemiol 2014;67:401-9.

33 Hoomans T, Severens JL. Economic evaluation of implementation strategies in health care: Biomed central. 9, 2014. 